\title{
Recurrence of distal cholangiocarcinoma after R0 resection: Differing survival outcomes between the locoregional vs. distant recurrence
}

\author{
Incheon KANG*
}

Department of Surgery, CHA Bundang Medical Center, CHA University School of Medicine, Korea

Introduction: The aim of this retrospective study was to investigate whether subtype of recurrence pattern affects the long-term outcome after curative surgical resection in patients with distal cholangiocarcinoma (DCC).

Methods: In total, patients with DCC who underwent pancreaticoduodenectomy were evaluated for recurrence. Patients with recurrence were classified into 2 groups according to the initial recurrence site: locoregional (LRR) and distant (DR). Clinicopathologic features were compared between 2 groups (LRR vs. DR), whereas overall survival (OS) after resection was analyzed among 3 groups (no recurrence [NR] vs. LRR vs DR). Prognostic factors for disease-free survival (DFS) and OS were also evaluated using the Cox proportional hazards model.

Results: A total of 214 patients underwent R0 surgical resection for DCC, of whom 109 (50.9\%) patients had recurred in the follow-up period. Of the 109 patients, 37 (33.1\%) had LRR and 72 (66.9\%) had DR. DR group was associated with early recurrence (14.1 months vs. 20.2 months; $p=0.041$ ) and a lower number of harvested lymph nodes (15.1 vs. 21.6; $p=0.001)$ than the LR group. Five-year OS was $98.7 \%, 40.5 \%$, and $23.2 \%$ in the NR, LRR, and DR groups, respectively $(p<0.001)$. In multivariate analysis, perineural invasion and pathologic N2 stage were independent prognostic factors for DFS ( $p=0.044$ and $p=0.008$, respectively), whereas pathologic N1 and N2 stage were independent prognostic factors associated with OS.

Conclusions: Even in R0 resected patients with DCC, almost half of patients experienced disease recurrence. DR was associated with a more frequent recurrence pattern and poorer survival than LRR. 